\title{
Separation of Oil-Water Emulsion in Laboratory Setup with U-Shaped Elements
}

\author{
Dang Vinh ${ }^{1}$, Vadim Zinurov ${ }^{1}$, Oksana Dmitrieva ${ }^{2, *}$, and Vitaly Kharkov ${ }^{2,3}$ \\ ${ }^{1}$ Kazan State Power Engineering University, 420066, Krasnoselskaya street 51, Kazan, Russia \\ ${ }^{2}$ Kazan National Research Technological University, 420015, Karl Marx street 68, Kazan, Russia \\ ${ }^{3}$ Kazan National Research Technical University named after A. N. Tupolev - KAI, 420111, Karl \\ Marx street 10, Kazan, Russia
}

\begin{abstract}
This study considers the problem of separation of oil-water emulsion in the case of oil spills. The standard methods of separation of the emulsion were presented. A unit with U-shaped separation elements has been developed. The device's operating principle was described, in which the separation of the oil-water emulsion occurs mainly due to the action of centrifugal forces arising from flow motion between the U-shaped elements and gravitational forces. The laboratory setup used for experiments was presented. The aim was to study the separation process of oil-water emulsion in a setup under varying initial temperature and initial density of the emulsion. The results showed that the device can separate the oil-water emulsion into light and heavy phases with an efficiency of at least $93.4 \%$ at a velocity of $1.39-2.15 \mathrm{~m} / \mathrm{s}$ in narrow sections between the U-shaped elements. One way of improving the separation of the oil-water emulsion is to increase the working temperature. It was found that the increased efficiency of $2.5 \%$ indicates that device applicability caused the presence and availability of heating devices. As the crude oil content in the feed increases, it is necessary to increase the number of rows of U-shaped elements or repeat the process.
\end{abstract}

\section{Introduction}

Crude oil spills cause severe damage to aquatic ecosystems. Nowadays, an important task is to quickly and effectively clean-up contaminated waters from them because the consequences are tragic for natural processes, including changes in habitat conditions and losses for human society. Due to their lower density relative to water, oil products are floating on the surface of the sea or lake, forming an oil slick that changes the composition of the water and prevents the passage of oxygen through the oil layer, causing oxygen deficit. If water is not treated in the short term, then heavy oil products gradually begin to settle, contaminating benthal deposits. After a time, oil-water emulsions are formed, a separation of which becomes more difficult [1-3].

Moreover, oil products in water supply systems can have harmful economic, environmental, public health, and social effect. In particular, oil spills lead to a decrease in

\footnotetext{
*Corresponding author: ja_deva@mail.ru
} 
the population of diverse marine species, especially birds and marine invertebrates, mammals, to an increase in various diseases, including oncological and cardiovascular diseases in humans and other negative environmental consequences [4-7].

The challenge of primary importance when releasing oil products into freshwater or marine ecosystems is their localization. Containment booms or simply booms are ubiquitously used for this purpose [8], which typically come in three types:

- fence ones, as a rule, are utilized to reduce the possibility of polluting shorelines and nature protective zones;

- sorbent devices made of porous material, which absorbs the oil while it is being contained;

- inflatable booms made from a strong and high-visibility fabric are suitable for the initial oil slick containment.

Globally the following methods are common for removing oil from water [9, 10] thermal, mechanical, physicochemical, and biological. The thermal method consists of burning at the spill site or in the chamber, bubbler, rotary furnaces, etc. The mechanical method is a treatment of oil products by physical processes, from manual scooping to machine. The physicochemical method involves treating the oil layer with small thicknesses using surfactants (sorbents, dispersants, etc.). The biological method is based on the ability of some microorganisms to convert aromatic and aliphatic hydrocarbons into carbon dioxide and water. The capturing of oil products from the water surface is carried out by special technical devices - oil collectors, skimmers, which allow collecting the top layer of water with the oil for further clean-up.

The most promising method of clean-up water from oil products (separation of oil-water emulsion) is mechanical due to its low cost, high rate, and environmental friendliness compared with thermal methods. However, mechanical water treatment in most cases is preliminary since the efficiency varies in a wide range from 60 to $95 \%$. Note that the mechanical method can only be a single operation when the cleaned water is dumped into the reservoirs without environmental disruption. In other cases, mechanical removal of oil from water should be combined with other methods [11-14].

The most common devices used for the mechanical treatment of water from oil products are settling tanks, hydrocyclones, filters, etc. The simplest and widely applied devices for wastewater treatment are settling tanks, which come in static, dynamic, thin-layer, tubular. Under the action of gravity, heavy impurities settle to the bottom, and the light ones float on its surface. The main drawbacks of the settling tanks are the low separation rate for oilwater emulsion and the vast footprint (it takes up much space). For example, when using static settling tanks, water treatment from oil products and other impurities is carried out periodically, and settling time is taken from 6 to 24 hours. Therefore, the oil-water emulsion settling tanks are used extremely rarely in oil spills. An operating principle of hydrocyclones involves an occurrence of centrifugal forces that act on oil droplets and throw them to the periphery of the device. Further, oil products are discharged through the lower outlet of the hydrocyclone. One of the main disadvantages of hydrocyclones is their low efficiency of about $70 \%$.

Filters are the most effective devices for removing oil from water, based on the adhesion of coarse particles of oil products to the surface of the filter media. The main disadvantage of the filtration process is periodicity, as the filter elements are gradually contaminated and need to be replaced. Therefore, so far, an urgent task for cleaning up major oil spills is developing compact devices for separating oil-water emulsions with high efficiency [15-20].

To address this problem, a compact separation device with U-shaped elements was developed, which consists of an inlet 1 for oil-water emulsion supply, U-shaped elements 2, and holes for removal of oil products 3 , a body 4, and an outlet 5 (Fig. 1). Notice that U- 
shaped elements are positioned in staggered rows. At the same time, even rows of U-shaped elements can be cut in half to decrease in dimensions, which does not affect the flow pattern.

The operating principle of the separation device with U-shaped elements is as follows. Oil-water emulsion enters the device through inlet 1 and flows between rows of U-shaped elements 2. It is worth noting that the U-shaped elements are aligned with that when the liquid flows around them, centrifugal forces arise which acting on the flow and droplets, and the liquid is divided into two phases depending on their density, the oil components rush into the made holes. Often, the described process is disrupted since the centrifugal forces partially discard both the light and heavy phases, as a result of which the separation efficiency of the oil-water emulsion decreases.

In order to achieve the maximum centrifugal forces when the flow of liquid with oil components moves, it is necessary to fulfill the following structural condition: the auxiliary circle drawn from the center of the U-shaped element must pass through the extreme points of the protrusions of the U-shaped elements of neighboring rows. Thus, as the liquid with dispersed oil globules moves in the device, the oil fraction is separated by centrifugal and gravitational forces [21-24].

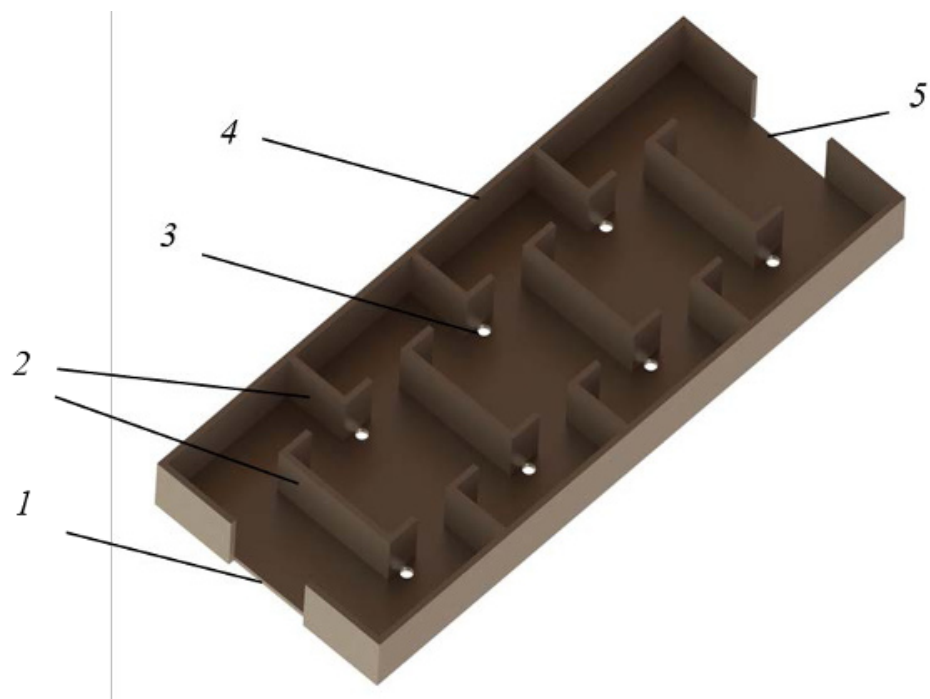

Fig. 1. Simplified 3D model of separation device with U-shaped elements (sectional view): 1 - inlet; 2 -U-shaped elements; 3 - holes for oil products discharge; 4 - device body; 5 - outlet.

A primary task in developing a novel device for the oil-water emulsion separation is their study under changing parameters (technological, thermophysical, and others) and identifying the most promising ways for intensifying the water purification from oil products. Therefore, the work aims to study the separation process of oil-water emulsion in a laboratory setup with U-shaped elements.

\section{Experimental methodology}

The laboratory setup used in this study includes a disperser (1) and a mixing tank (2), a pump 3, an apparatus 4 with installed inside U-shaped separation elements 5 (Fig. 2). 


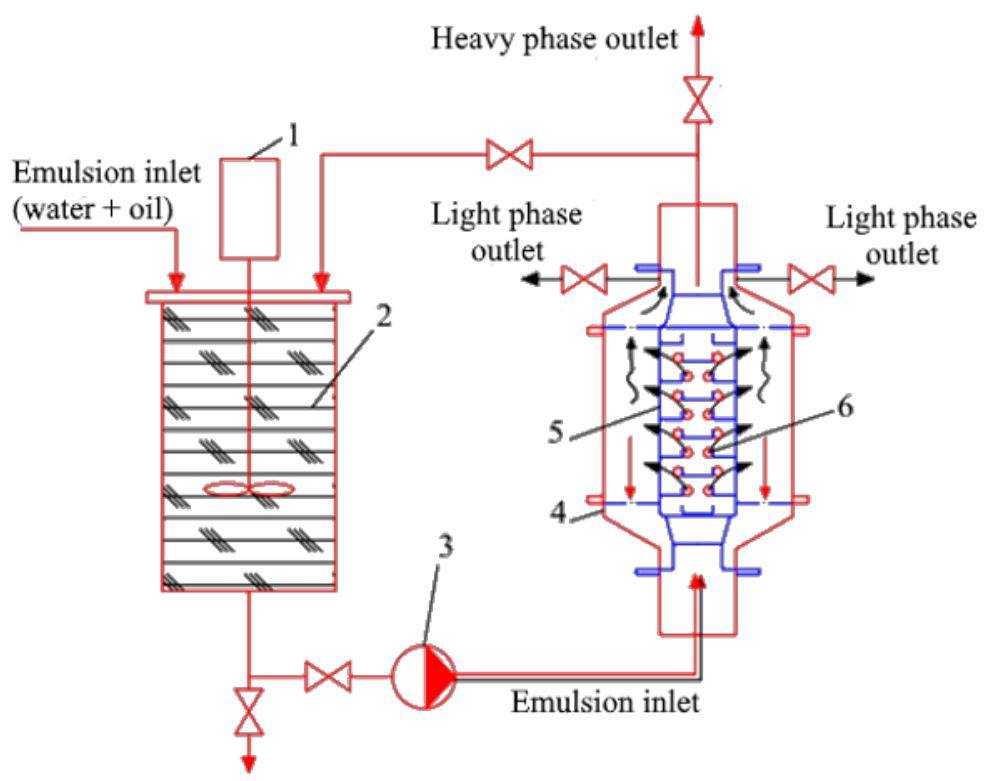

Fig. 2. Laboratory test setup with U-shaped separation elements: 1 - disperser; 2 - mixing tank; 3 pump; 4 - apparatus body; 5 - unit with U-shaped separation elements; 6 - holes of oil products discharge.

The experimental procedure was as follows. Water and oil were supplied into mixing tank 2, where using disperser 1, an oil-water emulsion was prepared. After mixing, the emulsion was pumped into apparatus 4 . When the oil-water mixture enters the test unit with the U-shaped separation elements 5, the separation process into the different density phases occurs as described above. It should be noted that the part of the oil-water emulsion which exited through the middle section of the outlet pipe from test unit 5 returns to the emulsion preparation tank 2. A ball valve was used to control the flow rate of the oil-water emulsion.

During the experiments, the densities of the initial emulsion, light and heavy phases were being determined using the AM MDS-300 areometer, used to measure the density of oil products from 800 to $1010 \mathrm{~kg} / \mathrm{m}^{3}$. The average weight flow ratio of heavy and light phases was 1:10. The velocity of the oil-water emulsion in the narrow sections of the unit with U-shaped separation elements was from 1.39 to $2.15 \mathrm{~m} / \mathrm{s}$. The initial temperature of the oil-water emulsion was $30^{\circ} \mathrm{C}$. The basic physicochemical properties of the crude oil used in the experiments are listed in Table 1 . The crude oil used in the studies had a high freezing point, about $33^{\circ} \mathrm{C}$, the content of solid paraffin in crude oil was $27 \%$, the melting point of solid paraffin was in the range from 50 to $65^{\circ} \mathrm{C}$.

Table 1. Chemical \& Physical Properties of Crude Oil.

\begin{tabular}{|c|c|c|}
\hline Parameter & Crude oil & Test method \\
\hline API & 38.6 & ASTM D 1298-96 \\
\hline Freezing point, ${ }^{\circ} \mathrm{C}$ & 33 & ASTM D 97; ASTM D 6749-02 \\
\hline Kinematic viscosity at $40^{\circ} \mathrm{C}, \mathrm{cSt}$ & 10 & ASTM D 445 \\
\hline Paraffin content, $\mathrm{mass} \%$ & 27 & GOST 11858-85 \\
\hline Asphaltenes content, $\mathrm{mass} \%$ & 0.77 & GOST 1185-85 (ASTM D 6560) \\
\hline Molecular weight, g/mol & 392 & ASTM D 2502 \\
\hline Acid number, $\mathrm{mg} \mathrm{KOH} / \mathrm{g}$ & 0.04 & ASTM D 664-89 (ASTM D 664) \\
\hline Water content, mass\% & 12 & ASTM D 95; (ASTM D 1744) \\
\hline
\end{tabular}


The effect of the content of the crude oil in the feed tank 2 and the temperature which varied from 30 to $75^{\circ} \mathrm{C}$ on the separation efficiency $E$ was examined by the following equation:

$$
E=\frac{\overline{x_{D}}-\overline{x_{F}}}{1-\overline{x_{F}}},
$$

where $\overline{x_{D}}$ is the mass fraction of oil in the light phase, $\mathrm{kg} / \mathrm{kg} ; \overline{x_{F}}$ is the mass fraction of oil in the feed emulsion, $\mathrm{kg} / \mathrm{kg}$.

\section{Results and discussion}

The results show that the device with U-shaped elements can separate oil-water emulsion with the efficiency in the range of $96.4-98.8 \%$ at an initial temperature of $30-75^{\circ} \mathrm{C}$ and an initial density of $848-996 \mathrm{~kg} / \mathrm{m}^{3}$, as shown in Fig. 3-4. A high velocity of oil-water emulsion flow inside the unit with separation elements - averaged value was of $1.7 \mathrm{~m} / \mathrm{s}$ at narrow sections between U-shaped elements. High velocities produced high centrifugal forces that lead to the formation of stable swirls at the round holes 3 (Fig. 1), resulting in an increase in the separation efficiency of the oil-water emulsion into light and heavy phases. The increase in the temperature of the feed emulsion promotes the separation process. At higher temperatures, the viscosity of the emulsion decreases, increasing the density difference between water and crude oil.

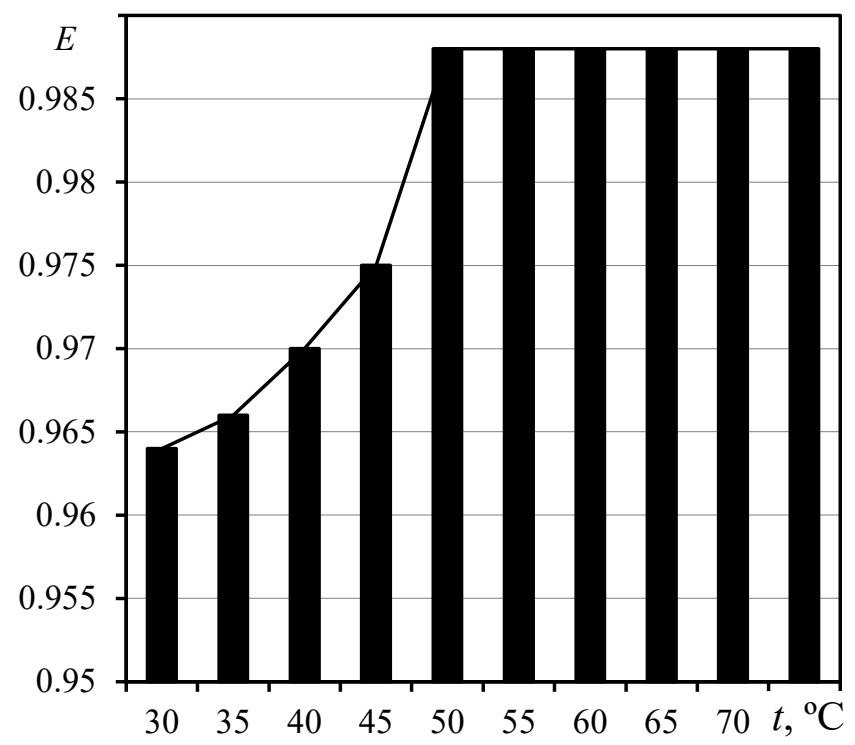

Fig. 3. Influence of initial temperature on separation efficiency.

It should be noted that with a rise in the temperature of the oil-water emulsion, the surface strength of the oil globules also decreases; as a consequence, their coalescence occurs. However, if this method is combined with chemical one, more additional research needs to be carried out since some demulsifiers are effective only at low temperatures, others only at high temperatures. The minimum separation efficiency of the oil-water emulsion was $96.4 \%$ at a feed temperature of $30^{\circ} \mathrm{C}$. As the temperature rises from 30 to $50^{\circ} \mathrm{C}$, the efficiency magnifies by $2.4 \%$. The constant separation efficiency of $98.8 \%$ was 
observed at a temperature above $50{ }^{\circ} \mathrm{C}$. The increase in the efficiency of the emulsion separation at temperatures of $30-75^{\circ} \mathrm{C}$ does not exceed $2.5 \%$, so this method can be recommended. Besides, when the temperature falls below $30^{\circ} \mathrm{C}$, there can be a significant decrease in the separation efficiency of the oil-water emulsion (Fig. 3).

An increase in the crude oil content in the feed promotes the growth of the density of the oil-water emulsion from 848 to $996 \mathrm{~kg} / \mathrm{m}^{3}$, causing a decrease in its separation efficiency from 98.8 to $93.4 \%$. The maximum separation efficiency is $98.8 \%$ when the density of the oil-water emulsion changes from 848 to $892 \mathrm{~kg} / \mathrm{m}^{3}$. The considerable decrease in emulsion separation efficiency from 98.8 to $93.7 \%$ occurs when its initial density varies from 892 to $976 \mathrm{~kg} / \mathrm{m}^{3}$. With an initial emulsion density of $976-996 \mathrm{~kg} / \mathrm{m}^{3}$, the separation efficiency is $93.5 \%$. At high oil content, the decline in the separation efficiency of the emulsion is observed, as some amount of the fractions are mixed again at the places of formation of swirls (near round holes). In order to improve efficiency, it is necessary to increase the number of rows of U-shaped elements (Fig. 4).

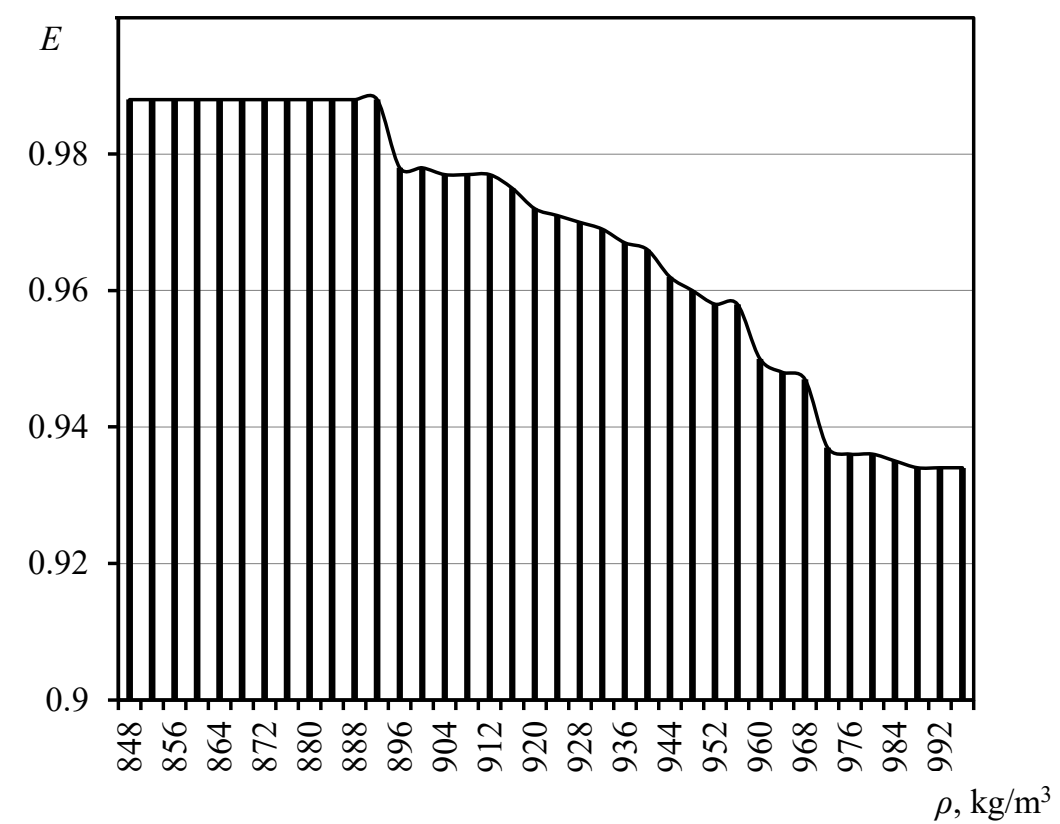

Fig. 4. Influence of initial density of oil-water emulsion on separation efficiency.

Thus, studies have shown that using the developed unit with U-shaped separation elements is a cost-efficient clean-up method for oil spills due to the high efficiency, compactness design, simplicity in operation, and the absence of moving parts. One promising way of improving the separation of the oil-water emulsion is to increase the working temperature. However, from obtained results, it is clear that the increased efficiency of $\pm 2.5 \%$ indicates that device applicability caused the presence and availability of heating devices. As the crude oil content in the feed increases, it is necessary to increase the number of rows of U-shaped elements or repeat the process.

\section{Conclusions}

The following conclusions can be drawn from the study: 
- the design of the separation device with the U-shaped elements has been developed, that provides the separation of the oil-water emulsion with the efficiency of more than $93.4 \%$ at its velocities from 1.39 to $2.15 \mathrm{~m} / \mathrm{s}$ in the narrow sections between the U-shaped elements;

- the separation process of the oil-water emulsion is mainly performed due to the action of centrifugal forces arising from the flow motion between the U-shaped elements;

- increase in the separation efficiency of the oil-water emulsion to $98.8 \%$ with increasing the temperature to $50^{\circ} \mathrm{C}$;

- reduction in the separation efficiency with increasing the concentration of crude oil in the feed mixture. When the density of the initial mixture varies from 848 to $996 \mathrm{~kg} / \mathrm{m}^{3}$, the separation efficiency decreases from 98.8 to $93.4 \%$ due to secondary mixing along the height of the device, where round holes are made;

- the main benefits of the developed separator with the U-shaped elements are compactness, high efficiency, and low operating costs.

The reported study was funded by the grant of the President of the Russian Federation, project number MK-616.2020.8.

\section{References}

1. M. D’Andrea and K. Reddy, Am. J. Med., 127 (2014).

2. G. M. Solomon and S. Janssen, JAMA, 304, 1118 (2010).

3. S. E. Chang, J. Stone, K. Demes, and M. Piscitelli, Ecol. Soc., 19, 26 (2014).

4. J. W. Farrington, Mar. Pollut. Bull., 150, 110744 (2020).

5. M. Lee, M. S. Park, and H. K. Cheong, Ecotoxicol. Environ. Saf., 194, 110284 (2020).

6. K. A. Colvin, C. Lewis, and T. S. Galloway, Chemosphere, 245, 125585 (2020).

7. F. Aguilera, J. Mendez, E. Pásaro, and B. Laffon, J. Appl. Toxicol., 30, 291 (2010).

8. M. Fingas, in (2021), pp. 875-889.

9. O. A. Johnson and A. C. Affam, Environ. Eng. Res., 24, 191 (2019).

10. A. Hoang, X. P. Nguyen, X.-Q. Duong, and T. Huynh, Environ. Sci. Pollut. Res., (2021).

11. R. K. Gupta, G. J. Dunderdale, M. W. England, and A. Hozumi, J. Mater. Chem. A, 5, 16025 (2017).

12. K. Wang, W. Yiming, J. Saththasivam, and Z. Liu, Nanoscale, 9, 9018 (2017).

13. A. Cambiella, J. M. Benito, C. Pazos, and J. Coca, Chem. Eng. Res. Des., 84, 69 (2006).

14. A. Dmitriev, V. Zinurov, O. Dmitrieva, and V. Dang Xuan, Her. Kazan State Power Eng. Univ., 3 (39), 65 (2018).

15. Q. Zeng, Z. Wang, X. Wang, Y. Zhao, and X. Guo, J. Pet. Sci. Eng., 145, 83 (2016).

16. A. Dmitriev, V. Zinurov, D. Vinh, and O. Dmitrieva, E3S Web Conf., 110, 1026 (2019).

17. X. Zeng, L. Zhao, G. Fan, and C. Yan, Chem. Eng. Res. Des., 165, 308 (2021).

18. S. Y. Shi, J. Y. Xu, H. Q. Sun, J. Zhang, D. H. Li, and Y. X. Wu, Chem. Eng. Res. Des., 90, 1652 (2012).

19. I. Madyshev, I. Sabanaev, V. Kharkov, L. Ganiev, and A. Dmitriev, MATEC Web Conf., 329, 3007 (2020). 
20. J. J. Slot, Development of a Centrifugal In-Line Separator for Oil-Water Flows, University of Twente, 2013.

21. O. S. Popkova, W. L. Nguyen, O. S. Dmitrieva, I. N. Madyshev, and A. N. Nikolaev, J. Phys. Conf. Ser., 1210, 012114 (2019).

22. V. V. Kharkov, J. Phys. Conf. Ser., 980, 012006 (2018).

23. A. V. Dmitriev, V. E. Zinurov, and O. S. Dmitrieva, MATEC Web Conf., 224, 02073 (2018).

24. V. E. Zinurov, N. Z. Dubkova, O. S. Popkova, and O. S. Dmitrieva, J. Phys. Conf. Ser., 1745, 012090 (2021). 